\title{
Plant and necrotrophic fungal pathogen interaction: mechanism and mode of action
}

\begin{abstract}
Necrotrophic fungal pathogens cause major losses to fruit, vegetable, and cereal crops annually and the economic impact is more than that of diseases caused by biotrophic pathogens. These pathogens are devastating because they kill as they colonize through production of cell wall-degrading enzymes and phytotoxins, obtaining nutrients for growth and reproduction from the dead plant cells. They explore a wide variety of virulence strategies and based on these the pathogens are classified into host-specific and broad hostrange necrotrophs. Plants are equipped with an immune system as a defense mechanism while the necrotrophic fungal pathogenic arsenal suppresses the immune responses for disease manifestation. Plant defense response involves the interplay of signaling molecules which include various phytohormones like jasmonic acid, ethylene, salicylic and abscisic acid which also serve as regulators of the immune response. Coordination at the transcriptional level of genes for the production of defense molecules including antimicrobial phytoalexins and pathogenesis-related proteins by transcription factors such as WRKY33 and ERF which are responsive to the signaling molecules has been observed. The roles for several important transcription factors already unveiled through studies of mutants in the model plant, Arabidopsis thaliana and some of the information translatable to crop plants. The present chapter shows the interconnection between cell wall integrity and the action of signaling molecules in the expression of defense-related genes. Moreover, the epigenetic mechanism through DNA and histone modification is also discussed.
\end{abstract}

Keyword: Necrotrophic fungi; Phytohormones; Pathogenesis-related proteins; Signaling molecules; Transcription factors; Epigenetic 